\title{
PANCREATITE AGUDA BILIAR NA INFÂNCIA
}

\section{ACUTE GALLSTONE PANCREATITIS IN CHILDHOOD}

\section{Flavio Augusto Menin ${ }^{1}$; Alexandre Petreca ${ }^{2}$; Roni Leonardo Teixeira, ACBC-SP ${ }^{2}$}

\section{INTRODUÇÃO}

A pancreatite é uma entidade clínica pouco freqüente na infância. Diferente dos adultos, as causas mais comuns em crianças incluem as infecções virais, por ascaris, medicamentosas, traumas e anomalias estruturais. Assim, a litíase biliar é incomum como causa de pancreatite entre as crianças ${ }^{1}$.

\section{RELATO DE CASO}

Menina, parda, 10 anos de idade, longilinea, pesando $47 \mathrm{Kg}$, com inicio de quadro de dor abdominal de forte intensidade e vômitos há 10 horas, admitida na emergência pediátrica, quando então, ao exame físico, apresentava-se corada, afebril e anictérica, com dor à palpação abdominal profunda, principalmente em andar superior, sem sinais de irritação peritoneal. Não apresenta historia pregressa de exposição a drogas, icterícia, eliminação de ascaris, vermifugação recente, parotidite recente, trauma abdominal, episódios álgicos anteriores ou hemopatia familiar.

O hemograma apresentava-se com17500 leucócitos (normal-10 mil), com predomínio de segmentados (85\%) sem desvio à esquerda, amilase - $923 \mathrm{~g} / \mathrm{dl}$, cálcio- $8.4 \mathrm{mg} / 100 \mathrm{ml}$, lípase $-526 \mathrm{mUI} / \mathrm{ml}$, TGO-26 mUI/ml, TGP $-32 \mathrm{mUI} / \mathrm{ml}$ ebilirrubinas total $(0,6)$ e frações (direta - 0,4 e indireta - 0,2) normais. Aultrasonografia mostrou colelitíase, colédoco com calibre nos limites superiores da normalidade $(0,4 \mathrm{~cm})$ (Figura 1) e pâncreas aumentado de volume (Figura 2). A tomografia abdominal com contraste oral apresentou pâncreas de contornos normais e dimensões aumentadas com parênquima discretamente heterogêneo, mas sem sinais de isquemia e/ou necrose (Figura 3). Eletroforese de hemoglobinas sem alterações.

Foi instituído jejum, hidratação venosa, antibioticoterapia e analgesia, sendo encaminhada para a unidade semi - intensiva. No $3^{\circ}$ dia de internação apresentou melhora clínica, com queda da amilase $(79 \mathrm{~g} / \mathrm{dl})$, sendo introduzida dieta leve hipogordurosa. No $7^{\circ}$ dia de internação teve alta hospitalar, com a operação programada (vídeocolecistectomia) para 18 dias depois. No $11^{\circ}$ dia após a alta, a criança apresentou nova crise de dor abdominal, de menor intensidade em relação 'a primeira, apresentando alteração somente dos níveis de amilase $(459 \mathrm{~g} / \mathrm{dl})$, sendo os de- mais exames laboratoriais normais. Ficou então, internada até o dia da operação.

Foi submetida à vídeocolecistectomia com colangiografia intra-operatoria, sem intercorrências. Teve alta no $1^{\circ}$ dia do pós-operatório, fazendo acompanhamento ambulatorial por um período de 12 meses, com alta em seguida.

\section{DISCUSSÃO}

A pancreatite aguda biliar é rara entre crianças (6 $-12 \%)$, sendo as causas mais comuns, parte de doenças

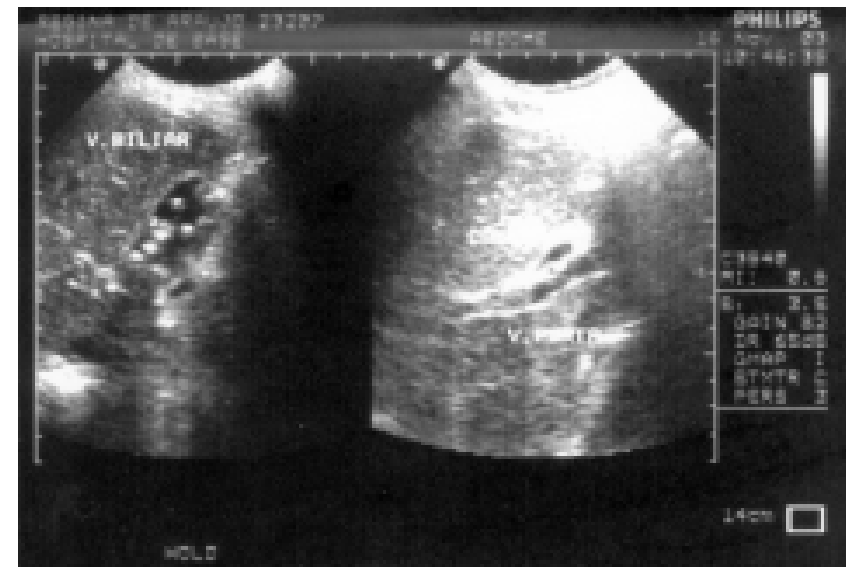

Figura 1 - Colelitíase e colédoco de calibre aumentado $(0,4 \mathrm{~cm})$.

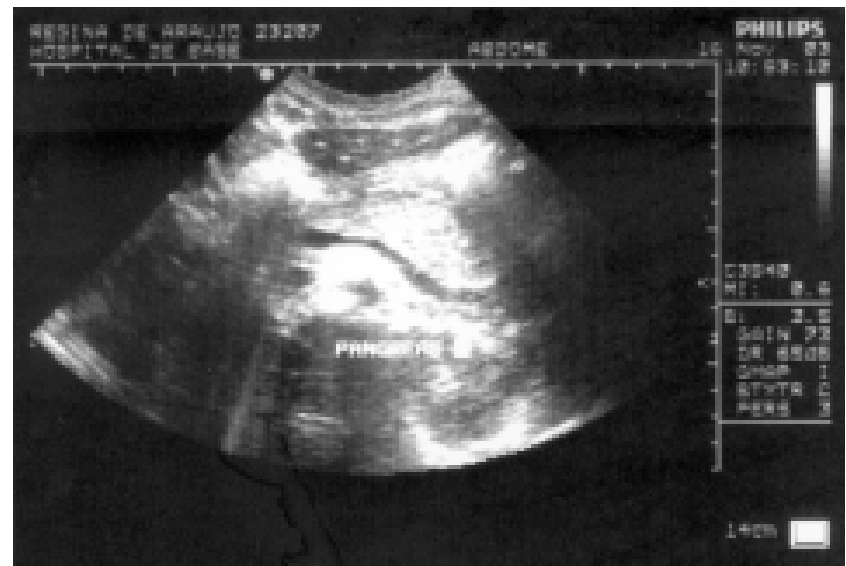

Figura 2 - Ultrassonografia mostrando pâncreas de dimensões aumentadas e ecotextura discretamente heterogênea.

1. Professor Colaborador da Disciplina de Cirurgia Pediátrica do Departamento de Pediatria e Cirurgia Pediátrica, da Faculdade de Medicina de São Jose do Rio Preto- SP.

2. Ex - Residente em Cirurgia Pediátrica do Hospital de Base da Faculdade de Medicina de São José do Rio Preto - SP.

Recebido em 28-05-2005

Aceito para publicação em 30-06-2005

Fonte de financiamento: nenhum

Conflito de interesses: nenhuma

Trabalho realizado no Hospital de Base da Faculdade de Medicina de São José do Rio Preto - SP 


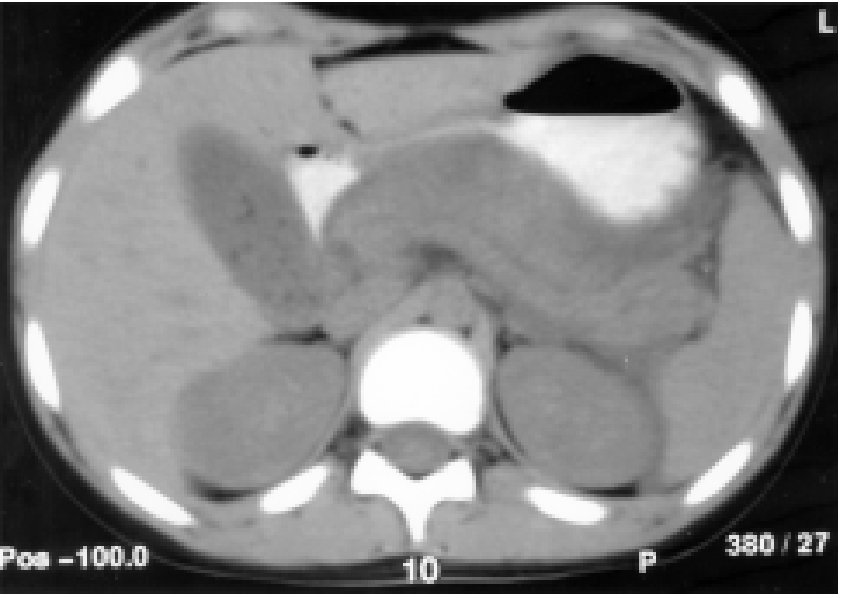

Figura 3 - Estudo tomográfico apresentando pâncreas de dimensões aumentadas com borramento da gordura peripancreática e líquido livre em cavidade.

multisistêmicas (35\%) tais como infecções virais, síndrome hemolítico urêmicas, choque hipovolêmico e/ou séptico; idiopática (30\%); e trauma (25\%). Alguns fatores predispõem à litíase na infância, tais como doença hemolítica crônica, obesidade, fibrose cística, ressecção ileal e doença hepática crônica. Em crianças, mais de 70 \% dos casos são cálculos pigmentados e 15 à $20 \%$ são de colesterol puro ${ }^{2}$.

A apresentação clínica da pancreatite biliar aguda na infância mostra uma menor incidência da forma grave do que nos adultos, especialmente em grupos bem jovens. A dor abdominal, que pode ser difusa, é mais freqüente de forma branda $(80 \%)$, seguido por vômitos em $50 \%$ dos casos ${ }^{2}$.
Como a apresentação clínica pode ser diversa e sutil, é necessário considerar o diagnóstico diferencial de pancreatite aguda na infância nestes casos. A amilase sérica está elevada em 83-90\% dos casos, além da lípase que persiste aumentada por um período maior, em relação à amilase ${ }^{1}$.

Os estudos radiológicos são acurados no diagnóstico de pancreatite. A ultrassonografia tem uma acurácia de aproximadamente $80 \%$ para pancreatite biliar. Já a tomografia computadorizada faz confirmação diagnóstica em $90 \%$ dos casos, além de analisar, em detalhes, o aumento focal ou difuso da glândula, bem como presença de processo inflamatório e/ou de abscessos, e estruturas adjacentes ${ }^{3}$. A colangiopancreatografia endoscópica retrógrada (CPRE) tem sido usada em larga escala entre os adultos, no entanto, este procedimento vem sendo adotado como modalidade terapêutica entre as crianças, onde, com mãos experientes, traz grandes benefícios à pacientes com pancreatite biliar e/ou colangite ${ }^{4}$.

A ressonância magnética colangiopancreatográfica é considerada equivalente à CPRE, para o diagnóstico da pancreatite e das doenças da árvore biliar, com sensibilidade em torno de 71-100\%, além de ser inócuo em relação à CPRE, porém, desvantajoso pelo alto custo ${ }^{5}$.

O tratamento de escolha do nosso serviço para litíase biliar é a colecistectomia vídeolaparoscópica, com colangiografia intra-operatoria, prevenindo episódios recorrentes de dor, icterícia e pancreatite. A presença de cálculos nos ductos biliares, nestes pacientes, está em torno de 2-6\%, em vigência de icterícia e/ou pancreatite.

O tempo de seguimento pós - operatório destes pacientes, é determinado pelo aparecimento de complicações, que, por ventura, este doente possa apresentar ${ }^{1,3,5}$.

\begin{abstract}
Acute pancreatitis is an uncommon condition in childhood. Gallstones rarely cause pancreatitis in children. Instead, the leading causes of pancreatitis tend to be trauma, infecctions, drugs, congenital disorders. One rare case of acute gallstone pancreatitis in children is described, showing the diagnosis clinical/radiologic and surgery treatment (videocolecistectomy) (Rev. Col. Bras. Cir. 2006; 33(5): 341-342).
\end{abstract}

Key words: Pancreatitis; Acute, diseases; Gallstones; Child.

\section{REFERÊNCIAS}

1. Calatayud GA, Bermejo F, Morales JL. Pancreatitis aguda en la infancia. Rev Esp Enferm Dig. 2003; 95(1):40-4.

2. Sutton R, Cheslyn-Curtis S. Acute gallstone pancreatitis in childhood. Ann R Coll Surg Engl. 2001; 83(6):406-8.

3. Goh SK, Chui CH, Jacobsen AS. Childhood acute pancreatitis in a children $\notin s$ hospital. Singapore Med J; 2003; 44(9):453-6.

4. Yachha SK, Chetri K, Saraswat VA. Management of childhood pancreatic disorders: a multidisciplinary approach.J Pediatr Gastroenterol Nutr. 2003; 36(2):206-12.

5. Jackson WD. Pancreatitis: etiology, diagnosis, and management. Curr Opin Pediatr. 2001; 13(5):447-51.
Como citar este artigo:

Menin FA, Petreca A, Teixeira RL. Pancreatite aguda biliar na infância: relato de caso. Rev Col Bras Cir. [periódico na Internet] 2006 Set-Out;33(5). Disponível em URL: www.scielo.br/rcbc

Endereço para correspondência:

Roni Leonardo Teixeira

Av. Tarumas, 2639- apto. 06

Res. Dona Sula

Jd. Maringá 2

78550-000 - SINOP - MT

E-mail: ronileonardo@zipmail.com.br 